\title{
Qualitative analysis for a delayed epidemic model with latent and breaking-out over the Internet
}

\section{Zizhen Zhang* and Yougang Wang}

\section{"Correspondence:}

zzzhaida@163.com

School of Management Science and

Engineering, Anhui University of

Finance and Economics, Bengbu,

233030, China

\begin{abstract}
We generalize a delayed computer virus model, known as the SLBQRS model in a computer network, by introducing the time delay due to the period that the antivirus software uses to clean viruses in the breaking out computers and the quarantined computers. By choosing the delay as the parameter, we prove the existence of a Hopf bifurcation as the delay crosses a critical value. Moreover, we study properties of the Hopf bifurcation by applying the center manifold theorem and the normal form theory. Finally, we carry out numerical simulations to support the obtained theoretical conclusions.
\end{abstract}

Keywords: computer virus; Hopf bifurcation; stability; SLBQRS model

\section{Introduction}

With the rapid development of the communication technology and network applications, the Internet has become an important platform for people sharing news and ideas [1]. Meanwhile, the Internet has become a powerful mechanism for propagating malicious computer virus programs such as worms, Trojans horses, and so on. What is more serious, enormous financial losses and social panic have also been caused by these computer viruses [2]. Therefore, it is urgent to understand the behavior of computer viruses and to pose effective measures of controlling their spread across the Internet.

In recent years, many models have been established to investigate spread of computer viruses since the pioneering work of Kephart and White [3]. They investigated the computer virus spreading in the Internet by using the SIS epidemic model inspired by the compelling similarities between computer viruses and their biological counterparts. The SIR classical epidemic model and some other different epidemic models are used to investigate the spread of malware in the Internet [4-7]. The authors of [8-11] proposed different SEIR models that assume that the recovered computers in networks have a permanent immunization period. SEIRS models and SEIQRS models have also been developed and studied in [12-15]. However, all the models mentioned overlook the fact that a computer can infect other computers immediately after it gets infected [16]. This is not consistent with realization. Because of that, an infected computer has infectivity during its latent period. Very recently, Kumar et al. [17] proposed the following computer virus model with 
graded infection rate based on the work by Yang et al. [18]:

$$
\left\{\begin{array}{l}
\frac{d S(t)}{d t}=\mu-\beta S(t)(L(t)+B(t))+\varepsilon R(t)-\mu S(t), \\
\frac{d L(t)}{d t}=\beta S(t)(L(t)+B(t))-(\mu+\alpha) L(t), \\
\frac{d B(t)}{d t}=\alpha L(t)-(\mu+\gamma+\eta+\sigma) B(t) \\
\frac{d Q(t)}{d t}=\gamma B(t)-(\mu+\sigma+\delta) Q(t) \\
\frac{d R(t)}{d t}=\delta Q(t)-(\mu+\varepsilon) R(t)+\eta B(t)
\end{array}\right.
$$

where $S(t), L(t), B(t), Q(t)$, and $R(t)$ denote the percentages of uninfected computers that have no immunity, infected computers that are latent, infected computers that are breaking out, infected computers that are quarantined, and recovered computers that have temporary immunity at time $t$, respectively; $\mu$ is the rate at which external computers are connected to the Internet, and it is also the rate at which the internal computers are disconnected from the Internet; $\sigma$ is the crashing rate of the computers due to the attack of computer viruses; and $\alpha, \beta, \gamma, \varepsilon, \eta$, and $\delta$ are the state transition rates of system (1).

It is well known that it needs some time to clean the computer viruses in the infected computers that are breaking out and in the infected computers that are quarantined for the anti-virus softwares. That is, system (1) neglects the delay due to the period that the anti-virus software needs to clean the viruses. To the best of our knowledge, until now there is no analysis on the dynamics of system (1) with time delay. Motivated by this fact, we consider the following delayed system:

$$
\left\{\begin{array}{l}
\frac{d S(t)}{d t}=\mu-\beta S(t)(L(t)+B(t))+\varepsilon R(t)-\mu S(t), \\
\frac{d L(t)}{d t}=\beta S(t)(L(t)+B(t))-(\mu+\alpha) L(t), \\
\frac{d B(t)}{d t}=\alpha L(t)-(\mu+\gamma+\eta+\sigma) B(t), \\
\frac{d Q(t)}{d t}=\gamma B(t)-(\mu+\sigma+\delta) Q(t), \\
\frac{d R(t)}{d t}=\delta Q(t-\tau)-(\mu+\varepsilon) R(t)+\eta B(t-\tau),
\end{array}\right.
$$

where $\tau$ is the delay due to the period that the antivirus software needs to clean the viruses. This paper mainly focuses on the effect of time delay on system (2).

The subsequent materials of this paper are organized as follows. In Section 2, we prove the local stability of the viral equilibrium and the existence of a Hopf bifurcation. Section 3 is devoted to investigations in direction of the Hopf bifurcation and stability of the bifurcating periodic solutions. Then, we validate the obtained results by using simulations in Section 4. We summarize this work in Section 5.

\section{Stability of viral equilibrium and existence of Hopf bifurcation}

According to the analysis in [19], we can obtain that system (2) has a unique viral equilib$\operatorname{rium} E_{*}\left(S_{*}, L_{*}, B_{*}, Q_{*}, R_{*}\right)$ if the basic reproduction number $R_{0}=\frac{\beta(\alpha+\gamma+\mu+\eta+\sigma)}{(\alpha+\mu)(\gamma+\mu+\eta+\sigma)}>1$, where

$$
\begin{aligned}
& S_{*}=\frac{(\alpha+\mu)(\gamma+\mu+\eta+\sigma)}{\beta(\alpha+\gamma+\mu+\eta+\sigma)}=\frac{1}{R_{0}}, \quad L_{*}=\frac{\gamma+\mu+\eta+\sigma}{\alpha} B_{*}, \\
& Q_{*}=\frac{\gamma}{\mu+\sigma+\delta} B_{*}, \quad R_{*}=\frac{\delta \gamma+\eta(\mu+\sigma+\delta)}{(\mu+\varepsilon)(\mu+\sigma+\delta)} B_{*},
\end{aligned}
$$




$$
B_{*}=\frac{\mu \alpha(\mu+\sigma+\delta)(\mu+\varepsilon)\left(1-R_{0}\right)}{R_{0} \alpha \gamma \delta \varepsilon+(\mu+\sigma+\delta)\left[R_{0} \alpha \eta \varepsilon-\beta(\mu+\varepsilon)(\mu+\alpha+\gamma+\eta+\sigma)\right]} .
$$

The linearized system of system (2) at the viral equilibrium $E_{*}\left(S_{*}, L_{*}, B_{*}, Q_{*}, R_{*}\right)$ is

$$
\left\{\begin{array}{l}
\frac{d S(t)}{d t}=a_{11} S(t)+a_{12} L(t)+a_{13} B(t)+a_{15} R(t), \\
\frac{d L(t)}{d t}=a_{21} S(t)+a_{22} L(t)+a_{23} B(t), \\
\frac{d B(t)}{d t}=a_{32} L(t)+a_{33} B(t), \\
\frac{d Q(t)}{d t}=a_{43} B(t)+a_{44} Q(t), \\
\frac{d R(t)}{d t}=a_{55} R(t)+b_{53} Q(t-\tau)+b_{54} B(t-\tau),
\end{array}\right.
$$

where

$$
\begin{aligned}
& a_{11}=-\beta\left(L_{*}+B_{*}\right)-\mu, \quad a_{12}=-\beta S_{*}, \quad a_{13}=-\beta S_{*}, \quad a_{15}=\varepsilon, \\
& a_{21}=\beta\left(L_{*}+B_{*}\right), \quad a_{22}=\beta S_{*}-(\mu+\alpha), \quad a_{23}=\beta S_{*}, \\
& a_{32}=\alpha, \quad a_{33}=\mu+\gamma+\sigma+\eta, \quad a_{43}=\gamma, \quad a_{44}=-(\mu+\sigma+\delta), \\
& a_{55}=-(\mu+\varepsilon), \quad b_{53}=\eta, \quad b_{54}=\delta .
\end{aligned}
$$

The characteristic equation of system (2) at $E_{*}\left(S_{*}, L_{*}, B_{*}, Q_{*}, R_{*}\right)$ can be obtained as follows:

$$
\lambda^{5}+p_{4} \lambda^{4}+p_{3} \lambda^{3}+p_{2} \lambda^{2}+p_{1} \lambda+p_{0}+\left(q_{1} \lambda+q_{0}\right) e^{-\lambda \tau}=0
$$

with

$$
\begin{aligned}
p_{0}= & a_{44} a_{55}\left[a_{33}\left(a_{12} a_{21}-a_{11} a_{22}\right)+a_{32}\left(a_{11} a_{23}-a_{13} a_{21}\right)\right], \\
p_{1}= & a_{55}\left[a_{11} a_{22}\left(a_{33}+a_{44}\right)+a_{33} a_{44}\left(a_{11}+a_{22}\right)\right]+a_{11} a_{22} a_{33} a_{44} \\
& +a_{13} a_{21} a_{32}\left(a_{44}+a_{55}\right)-a_{23} a_{32}\left[a_{11}\left(a_{44}+a_{55}\right)+a_{44} a_{55}\right] \\
& -a_{12} a_{21}\left[a_{33}\left(a_{44}+a_{55}\right)+a_{44} a_{55}\right], \\
p_{2}= & a_{23} a_{32}\left(a_{11}+a_{44}+a_{55}\right)+a_{12} a_{21}\left(a_{33}+a_{44}+a_{55}\right) \\
& -a_{13} a_{21} a_{32}-a_{11} a_{22}\left(a_{33}+a_{44}\right)-a_{33} a_{44}\left(a_{11}+a_{22}\right) \\
& -a_{55}\left[a_{11} a_{22}+a_{33} a_{44}+\left(a_{11}+a_{22}\right)\left(a_{33}+a_{44}\right)\right], \\
p_{3}= & a_{11} a_{22}+a_{33} a_{44}+a_{55}\left(a_{11}+a_{22}+a_{33}+a_{44}\right) \\
& +\left(a_{11}+a_{22}\right)\left(a_{33}+a_{44}\right)-a_{12} a_{21}-a_{23} a_{32}, \\
p_{4}= & -\left(a_{11}+a_{22}+a_{33}+a_{44}+a_{55}\right), \\
q_{0}= & a_{15} a_{32}\left(a_{44} b_{53}-a_{43} b_{54}\right), \quad q_{1}=-a_{15} a_{32} b_{53} .
\end{aligned}
$$

When $\tau=0$, equation (4) becomes

$$
\lambda^{5}+p_{4} \lambda^{4}+p_{3} \lambda^{3}+p_{2} \lambda^{2}+\left(p_{1}+q_{1}\right) \lambda+p_{0}+q_{0}=0 .
$$


Suppose that the following inequalities are satisfied, which we refer to as condition $\left(H_{1}\right)$ :

$$
\begin{aligned}
\operatorname{det}_{1} & =p_{4}>0, \\
\operatorname{det}_{2} & =\left(\begin{array}{cc}
p_{4} & 1 \\
p_{2} & p_{3}
\end{array}\right)>0, \\
\operatorname{det}_{3} & =\left(\begin{array}{ccc}
p_{4} & 1 & 0 \\
p_{2} & p_{3} & p_{4} \\
0 & p_{1}+q_{1} & p_{2}
\end{array}\right)>0, \\
\operatorname{det}_{4} & =\left(\begin{array}{cccc}
p_{4} & 1 & 0 & 0 \\
p_{2} & p_{3} & p_{4} & 1 \\
p_{0}+q_{0} & p_{1}+q_{1} & p_{12} & p_{3} \\
0 & 0 & p_{0}+q_{0} & p_{1}+q_{1}
\end{array}\right)>0, \\
\operatorname{det}_{5} & =\left(\begin{array}{ccccc}
p_{4} & 1 & 0 & 0 & 0 \\
p_{2} & p_{3} & p_{4} & 1 & 0 \\
p_{0}+q_{0} & p_{1}+q_{1} & p_{2} & p_{3} & p_{4} \\
0 & 0 & p_{0}+q_{0} & p_{1}+q_{1} & p_{2} \\
0 & 0 & 0 & 0 & p_{0}+q_{0}
\end{array}\right)>0 .
\end{aligned}
$$

Then $E_{*}\left(S_{*}, L_{*}, B_{*}, Q_{*}, R_{*}\right)$ is locally asymptotically stable.

For $\tau>0$, let $\lambda=i \omega(\omega>0)$ be a root of equation (4). Then,

$$
\left\{\begin{array}{l}
q_{1} \omega \sin \tau \omega+q_{0} \cos \tau \omega=p_{2} \omega^{2}-p_{4} \omega^{4}-p_{0} \\
q_{1} \omega \cos \tau \omega-q_{0} \sin \tau \omega=p_{3} \omega^{3}-\omega^{5}-p_{1} \omega
\end{array}\right.
$$

It follows that

$$
\omega^{10}+e_{4} \omega^{8}+e_{3} \omega^{6}+e_{2} \omega^{4}+e_{1} \omega^{2}+p_{0}=0
$$

where

$$
\begin{aligned}
& e_{0}=p_{0}^{2}-q_{0}^{2}, \quad e_{1}=p_{1}^{2}-2 p_{0} p_{2}-q_{1}^{2}, \\
& e_{2}=p_{2}^{2}+2 p_{0} p_{4}-2 p_{1} p_{3}, \quad e_{3}=p_{3}^{2}+2 p_{1}-2 p_{2} p_{4}, \quad e_{4}=p_{4}^{2}-2 p_{3} .
\end{aligned}
$$

Letting $\omega^{2}=v$, equation (12) becomes

$$
v^{5}+e_{4} v^{4}+e_{3} v^{3}+e_{2} v^{2}+e_{1} v+e_{0}=0
$$

The discussion of the distribution of positive real roots of equation (13) is similar to that in [20]. Obviously, if $e_{0}<0$, then equation (13) has at least one positive root. In the following, we discuss the distribution of the roots of equation (13) as $e_{0} \geq 0$.

Denote

$$
h(v)=v^{5}+e_{4} v^{4}+e_{3} v^{3}+e_{2} v^{2}+e_{1} v+e_{0} .
$$


Then,

$$
h^{\prime}(v)=5 v^{4}+4 e_{4} v^{3}+3 e_{3} v^{2}+2 e_{2} v+e_{1} .
$$

Define

$$
5 v^{4}+4 e_{4} v^{3}+3 e_{3} v^{2}+2 e_{2} v+e_{1}=0 .
$$

Let $v=z-\frac{e_{4}}{5}$. Then, equation (16) becomes

$$
z^{4}+c_{2} z^{2}+c_{1} z+c_{0}=0
$$

with

$$
\begin{aligned}
& c_{0}=-\frac{3}{625} e_{4}^{4}+\frac{3}{125} e_{4}^{2} e_{3}-\frac{2}{25} e_{4} e_{2}+\frac{1}{5} e_{1}, \\
& c_{1}=\frac{8}{125} e_{4}^{3}+\frac{6}{25} e_{4} e_{3}+\frac{2}{3} e_{2}, \\
& c_{2}=-\frac{6}{25} e_{4}^{2}+\frac{3}{5} e_{3} .
\end{aligned}
$$

If $c_{1}=0$, then we can obtain the four roots of equation (17) as follows:

$$
\begin{array}{ll}
z_{1}=\sqrt{\frac{-c_{2}+\sqrt{\triangle_{0}}}{2}}, & z_{2}=-\sqrt{\frac{-c_{2}+\sqrt{\triangle_{0}}}{2}}, \\
z_{3}=\sqrt{\frac{-c_{2}-\sqrt{\triangle_{0}}}{2}}, & z_{4}=-\sqrt{\frac{-c_{2}-\sqrt{\triangle_{0}}}{2}},
\end{array}
$$

with $\triangle_{0}=c_{2}^{2}-4 c_{0}$. Thus, $v_{i}=z_{i}-\frac{e_{4}}{5}(i=1,2,3,4)$ are the roots of equation (16). Then we have the following result.

Lemma 1 Suppose that $e_{0} \geq 0$ and $c_{1}=0$.

(i) If $\triangle_{0}<0$, then equation (13) has no positive real roots;

(ii) If $\triangle_{0} \geq 0, c_{2} \geq 0$, and $c_{0}>0$, then equation (13) has no positive real roots;

(iii) If (i) and (ii) are not satisfied, then equation (13) has positive real roots if and only if there exists at least one $v^{*} \in\left\{v_{1}, v_{2}, v_{3}, v_{4}\right\}$ such that $v^{*}>0$ and $h\left(v^{*}\right) \leq 0$.

Next, we assume that $c_{1} \neq 0$. Consider the resolvent of equation (17)

$$
c_{1}^{2}-4\left(s-c_{2}\right)\left(\frac{s^{2}}{4}-c_{0}\right)=0,
$$

that is,

$$
s^{3}-c_{2} s^{2}-4 c_{0} s_{1}+4 c_{2} c_{0}-c_{1}^{2}=0 .
$$

Denote

$$
\alpha_{1}=\frac{1}{3} c_{2}^{2}-4 c_{0}, \quad \beta_{1}=-\frac{2}{27} c_{2}^{3}+\frac{8}{3} c_{2} c_{0}-c_{1}^{2},
$$




$$
\triangle_{1}=\frac{1}{27} \alpha_{1}^{3}+\frac{1}{4} \beta_{1}^{2}, \quad \sigma_{1}=\frac{1}{2}+\frac{\sqrt{3}}{2} i
$$

By the Cardan formula equation (17) has the following three roots:

$$
\begin{aligned}
& s_{1}=\sqrt[3]{-\frac{\alpha_{1}}{2}+\sqrt{\triangle_{1}}}+\sqrt[3]{-\frac{\alpha_{1}}{2}-\sqrt{\triangle_{1}}+\frac{c_{2}}{3}}, \\
& s_{2}=\sigma_{1} \sqrt[3]{-\frac{\alpha_{1}}{2}+\sqrt{\triangle_{1}}}+\sigma_{1}^{2} \sqrt[3]{-\frac{\alpha_{1}}{2}-\sqrt{\triangle_{1}}}+\frac{c_{2}}{3}, \\
& s_{3}=\sigma_{1}^{2} \sqrt[3]{-\frac{\alpha_{1}}{2}+\sqrt{\triangle_{1}}}+\sigma_{1} \sqrt[3]{-\frac{\alpha_{1}}{2}-\sqrt{\triangle_{1}}}+\frac{c_{2}}{3} .
\end{aligned}
$$

Let $s_{*}=s_{1} \neq c_{2}$. Then equation (17) is equivalent to

$$
z^{4}+s_{*} z^{2}+\frac{s_{*}^{2}}{4}-\left[\left(s_{*}-c_{2}\right) z^{2}-c_{1} z+\frac{s_{*}^{2}}{4}-c_{0}\right]=0 .
$$

If $s_{*}>c_{2}$, then equation (20) is

$$
\left(z^{2}+\frac{s_{*}}{2}\right)^{2}-\left(\sqrt{s_{*}-c_{2}} z-\frac{c_{1}}{2 \sqrt{s_{*}-c_{2}}}\right)^{2}=0 .
$$

After factorization, we obtain

$$
z^{2}+\sqrt{s_{*}-c_{2}} z-\frac{c_{1}}{2 \sqrt{s_{*}-c_{2}}}+\frac{s_{*}}{2}=0
$$

and

$$
z^{2}-\sqrt{s_{*}-c_{2}} z+\frac{c_{1}}{2 \sqrt{s_{*}-c_{2}}}+\frac{s_{*}}{2}=0 .
$$

Denote

$$
\triangle_{2}=-s_{*}-c_{2}+\frac{2 c_{1}}{\sqrt{s_{*}-c_{2}}}, \quad \triangle_{3}=-s_{*}-c_{2}-\frac{2 c_{1}}{\sqrt{s_{*}-c_{2}}} .
$$

Then, we can obtain the roots of equation (17):

$$
\begin{aligned}
& z_{1}=\frac{-\sqrt{s_{*}-c_{2}}+\sqrt{\triangle_{2}}}{2}, \quad z_{2}=\frac{-\sqrt{s_{*}-c_{2}}-\sqrt{\triangle_{2}}}{2}, \\
& z_{3}=\frac{\sqrt{s_{*}-c_{2}}+\sqrt{\triangle_{3}}}{2}, \quad z_{4}=\frac{\sqrt{s_{*}-c_{2}}-\sqrt{\triangle_{3}}}{2} \text {. }
\end{aligned}
$$

Then, $v_{i}=z_{i}-\frac{e_{4}}{5}(i=1,2,3,4)$ are the roots of equation (16). Thus, we have the following result.

Lemma 2 Suppose that $e_{0} \geq 0, c_{1} \neq 0$, and $s_{*}>c_{2}$.

(i) If $\triangle_{2}<0$ and $\Delta_{3}<0$, then equation (13) has no positive real roots;

(ii) If (i) is not satisfied, then equation (13) has positive real roots if and only if there exists at least one $v^{*} \in\left\{v_{1}, v_{2}, v_{3}, v_{4}\right\}$ such that $v^{*}>0$ and $h\left(v^{*}\right) \leq 0$. 
Finally, if $s_{*}<c_{2}$, then equation (20) is

$$
\left(z^{2}+\frac{s_{*}}{2}\right)^{2}-\left(\sqrt{c_{2}-s_{*}} z-\frac{c_{1}}{2 \sqrt{c_{2}-s_{*}}}\right)^{2}=0 .
$$

Denote $\bar{v}=\frac{c_{1}}{2\left(c_{2}-s_{*}\right)}-\frac{e_{4}}{5}$. Hence, we have the following result.

Lemma 3 Suppose that $e_{0} \geq 0, c_{1} \neq 0$, and $s_{*}<c_{2}$. Then equation (13) has positive real roots if and only if $\frac{c_{1}^{2}}{4\left(c_{2}-s_{*}\right)^{2}}+\frac{s_{*}}{2}=0$ and $\bar{v}>0, h(\bar{v}) \leq 0$.

In conclusion, we can obtain that equation (13) has at one positive real root if the coefficients in equation (13) satisfy one of the conditions in $\left(H_{2}\right)$ :

$\left(H_{2}\right)$ (a) $e_{0}<0$; (b) $e_{0} \geq 0, c_{1}=0$, and $c_{2}<0$ or $c_{0}>0$, and there exists at least one $v^{*} \in$ $\left\{v_{1}, v_{2}, v_{3}, v_{4}\right\}$ such that $v^{*}>0$ and $h\left(v^{*}\right) \leq 0$; (c) $e_{0} \geq 0, c_{1} \neq 0, s_{*}>c_{2}, \Delta_{2} \geq 0$, or $\triangle_{3} \geq 0$, and there exists at least one $v^{*} \in\left\{v_{1}, v_{2}, v_{3}, v_{4}\right\}$ such that $v^{*}>0$ and $h\left(v^{*}\right) \leq 0$; (d) $e_{0} \geq 0, c_{1} \neq 0, s_{*}<c_{2}, \frac{c_{1}^{2}}{4\left(c_{2}-s_{*}\right)^{2}}+\frac{s_{*}}{2}=0$, and $\bar{v}>0, h(\bar{v}) \leq 0$.

Suppose that the coefficients in equation (13) satisfy one of the conditions in $\left(\mathrm{H}_{2}\right)$. Then equation (13) has at least one positive real root $v_{0}$ such that equation (4) has a pair of purely imaginary roots $\pm i \omega_{0}= \pm i \sqrt{v_{0}}$. For $\omega_{0}$, we have

$$
\tau_{0}=\frac{1}{\omega_{0}} \arccos \left\{\frac{-q_{1} \omega_{0}^{6}+\left(p_{3} q_{1}-p_{4} q_{0}\right) \omega_{0}^{4}+\left(p_{2} q_{0}-p_{1} q_{1}\right) \omega_{0}^{2}-p_{0} q_{0}}{q_{0}^{2}+q_{1}^{2} \omega_{0}^{2}}\right\}
$$

Differentiating equation (4) with respect to $\tau$ and using equation (4), we get

$$
\left[\frac{d \lambda}{d \tau}\right]^{-1}=-\frac{5 \lambda^{4}+4 p_{4} \lambda^{3}+3 p_{3} \lambda^{2}+2 p_{2} \lambda+p_{1}}{\lambda\left(\lambda^{5}+p_{4} \lambda^{4}+p_{3} \lambda^{3}+p_{2} \lambda^{2}+p_{1} \lambda+p_{0}\right)}+\frac{q_{1}}{\lambda\left(q_{1} \lambda+q_{0}\right)}-\frac{\tau}{\lambda} .
$$

Substituting $\lambda=i \omega_{0}$ into equation (26) and taking its real part, we have

$$
\operatorname{Re}\left[\frac{d \lambda}{d \tau}\right]_{\tau=\tau_{0}}^{-1}=\frac{h^{\prime}\left(v_{*}\right)}{q_{0}^{2}+q_{1}^{2} \omega_{0}^{2}}
$$

where $v_{*}=\omega_{0}^{2}$.

Obviously, if condition

$\left(H_{3}\right) \quad h^{\prime}\left(\omega_{0}^{2}\right) \neq 0$

holds, then $\operatorname{Re}\left[\frac{d \lambda}{d \tau}\right]_{\tau=\tau_{0}}^{-1} \neq 0$. Thus, we can conclude that if condition $\left(H_{3}\right)$ holds, then the transversality condition is satisfied. According to the Hopf bifurcation theorem in [19], we have the following:

Theorem 1 For system (2), if conditions $\left(H_{1}\right)-\left(H_{3}\right)$ hold, then the viral equilibrium $E_{*}\left(S_{*}, L_{*}, B_{*}, Q_{*}, R_{*}\right)$ is asymptotically stable for $\tau \in\left[0, \tau_{0}\right)$. A Hopf bifurcation occurs at the viral equilibrium $E_{*}\left(S_{*}, L_{*}, B_{*}, Q_{*}, R_{*}\right)$ when $\tau=\tau_{0}$, and a family of periodic solutions bifurcate from the viral equilibrium $E_{*}\left(S_{*}, L_{*}, B_{*}, Q_{*}, R_{*}\right)$ near $\tau=\tau_{0}$. 


\section{Stability of the bifurcating periodic solutions}

Let $u_{1}(t)=S(t)-S_{*}, u_{2}(t)=L(t)-L_{*}, u_{3}(t)=B(t)-B_{*}, u_{4}(t)=Q(t)-Q_{*}, u_{5}(t)=R(t)-$ $R_{*}, \tau=\tau_{0}+\mu, \mu \in R$. By the transformation $t=t / \tau$ system (2) becomes the following functional differential equation in $C=C\left([-1,0], R^{5}\right)$ :

$$
\dot{u}(t)=L_{\mu} u_{t}+F\left(\mu, u_{t}\right)
$$

where $u_{t}=\left(u_{1}(t), u_{2}(t), u_{3}(t), u_{4}(t), u_{5}(t)\right)^{T}=(S(t), L(t), B(t), Q(t), R(t))^{T} \in R^{5}, u_{t}(\theta)=u(t+$ $\theta) \in C$, and $L_{\mu}: C \rightarrow R^{5}$ and $F\left(\mu, u_{t}\right) \rightarrow R^{5}$ are defined by

$$
L_{\mu} \phi=\left(\tau_{0}+\mu\right)\left(\begin{array}{ccccc}
a_{11} & a_{12} & a_{13} & 0 & a_{15} \\
a_{21} & a_{22} & 0 & 0 & 0 \\
0 & a_{32} & a_{33} & 0 & 0 \\
0 & 0 & a_{43} & a_{44} & 0 \\
0 & 0 & 0 & 0 & a_{55}
\end{array}\right) \phi(0)+\left(\tau_{0}+\mu\right)\left(\begin{array}{ccccc}
0 & 0 & 0 & 0 & 0 \\
0 & 0 & 0 & 0 & 0 \\
0 & 0 & 0 & 0 & 0 \\
0 & 0 & 0 & 0 & 0 \\
0 & 0 & b_{53} & b_{54} & 0
\end{array}\right) \phi(-1)
$$

and

$$
F(\mu, \phi)=\left(\begin{array}{c}
-\beta\left(\phi_{1}(0) \phi_{2}(0)+\phi_{1}(0) \phi_{3}(0)\right) \\
\beta\left(\phi_{1}(0) \phi_{2}(0)+\phi_{1}(0) \phi_{3}(0)\right) \\
0 \\
0 \\
0
\end{array}\right)
$$

By the Riesz representation theorem there is a bounded-variation function $\eta(\theta, \mu)$ in $\theta \in[-1,0]$ such that

$$
L_{\mu} \phi=\int_{-1}^{0} d \eta(\theta, \mu) \phi(\theta) \quad \text { for } \phi \in C .
$$

In fact, we choose

$$
\begin{aligned}
& \eta(\theta, \mu)=\left(\tau_{0}+\mu\right)\left(\begin{array}{ccccc}
a_{11} & a_{12} & a_{13} & 0 & a_{15} \\
a_{21} & a_{22} & 0 & 0 & 0 \\
0 & a_{32} & a_{33} & 0 & 0 \\
0 & 0 & a_{43} & a_{44} & 0 \\
0 & 0 & 0 & 0 & a_{55}
\end{array}\right) \delta(\theta) \\
&+\left(\tau_{0}+\mu\right)\left(\begin{array}{ccccc}
0 & 0 & 0 & 0 & 0 \\
0 & 0 & 0 & 0 & 0 \\
0 & 0 & 0 & 0 & 0 \\
0 & 0 & 0 & 0 & 0 \\
0 & 0 & b_{53} & b_{54} & 0
\end{array}\right) \delta(\theta+1),
\end{aligned}
$$

where $\delta(\theta)$ is the Dirac delta function.

For $\phi \in C\left([-1,0], R^{5}\right)$, we set

$$
A(\mu) \phi= \begin{cases}\frac{d \phi(\theta)}{d \theta}, & -1 \leq \theta<0, \\ \int_{-1}^{0} d \eta(\theta, \mu) \phi(\theta), & \theta=0\end{cases}
$$


and

$$
R(\mu) \phi= \begin{cases}0, & -1 \leq \theta<0 \\ F(\mu, \phi), & \theta=0\end{cases}
$$

Then system (27) can be rewritten as

$$
\dot{u}(t)=A(\mu) u_{t}+R(\mu) u_{t}
$$

For $\varphi \in C^{1}([0,1]),\left(R^{5}\right)^{*}$, we define the operator

$$
A^{*}(\varphi)= \begin{cases}-\frac{d \varphi(s)}{d s}, & 0<s \leq 1 \\ \int_{-1}^{0} d \eta^{T}(s, 0) \varphi(-s), & s=0\end{cases}
$$

and the bilinear inner form

$$
\langle\varphi(s), \phi(\theta)\rangle=\bar{\varphi}(0) \phi(0)-\int_{\theta=-1}^{0} \int_{\xi=0}^{\theta} \bar{\varphi}(\xi-\theta) d \eta(\theta) \phi(\xi) d \xi
$$

where $\eta(\theta)=\eta(\theta, 0)$.

Then, it is easy to see that $A(0)$ and $A^{*}(0)$ are adjoint operators and that $\pm i \omega_{0}$ are the eigenvalues of $A(0)$ and also the eigenvalues of $A^{*}(0)$.

Let $\rho(\theta)=\left(1, \rho_{2}, \rho_{3}, \rho_{4}, \rho_{5}\right)^{T} e^{i \omega_{0} \tau_{0} \theta}$ and $\rho^{*}(s)=\left(1, \rho_{2}^{*}, \rho_{3}^{*}, \rho_{4}^{*}, \rho_{5}^{*}\right) e^{i \omega_{0} \tau_{0} s}$ be the eigenvectors of $A(0)$ and $A^{*}(0)$ corresponding to $+i \omega_{0} \tau_{0}$, respectively. Then, it is not difficult to show that

$$
\begin{aligned}
& \rho_{2}=\frac{i \omega_{0}-a_{33}}{a_{32}} \rho_{3}, \quad \rho_{3}=\frac{a_{21} a_{32}}{\left(i \omega_{0}-a_{22}\right)\left(i \omega_{0}-a_{33}\right)-a_{23} a_{32}}, \\
& \rho_{4}=\frac{a_{43} \rho_{3}}{i \omega_{0}-a_{44}}, \quad \rho_{5}=\frac{b_{53} \rho_{3}+b_{54} \rho_{4}}{\left(i \omega_{0}-a_{33}\right) e^{i \tau_{0} \omega_{0}}}, \\
& \rho_{2}^{*}=-\frac{i \omega_{0}+a_{11}}{a_{21}}, \quad \rho_{3}^{*}=-\frac{a_{12}+\left(i \omega_{0}+a_{22}\right) \rho_{2}^{*}}{a_{32}}, \\
& \rho_{4}^{*}=\frac{a_{15} b_{54} e^{i \tau_{0} \omega_{0}}}{\left(i \omega_{0}+a_{44}\right)\left(i \omega_{0}+a_{55}\right)}, \quad \rho_{5}^{*}=-\frac{a_{15}}{i \omega_{0}+a_{55}} .
\end{aligned}
$$

Using equation (30), we can obtain

$$
\left\langle\rho^{*}(s), \rho(\theta)\right\rangle=\bar{D}\left[1+\rho_{2} \bar{\rho}_{2}^{*}+\rho_{3} \bar{\rho}_{3}^{*}+\rho_{4} \bar{\rho}_{4}^{*}+\rho_{5} \bar{\rho}_{5}^{*}+\tau_{0} e^{-i \tau_{0} \omega_{0}} \rho_{5}^{*}\left(b_{53} \rho_{3}+b_{54} \rho_{4}\right)\right] \text {. }
$$

Then we choose

$$
\bar{D}=\left[1+\rho_{2} \bar{\rho}_{2}^{*}+\rho_{3} \bar{\rho}_{3}^{*}+\rho_{4} \bar{\rho}_{4}^{*}+\rho_{5} \bar{\rho}_{5}^{*}+\tau_{0} e^{-i \tau_{0} \omega_{0}} \rho_{5}^{*}\left(b_{53} \rho_{3}+b_{54} \rho_{4}\right)\right]^{-1}
$$

such that $\left\langle\rho^{*}, \rho\right\rangle=1$ and $\left\langle\rho^{*}, \bar{\rho}\right\rangle=0$. 
Following the method introduced in [19] and using a similar computation process in [21-23], we can get the following coefficients:

$$
\begin{aligned}
g_{20}= & 2 \tau_{0} \bar{D} \beta\left(\bar{\rho}_{2}^{*}-1\right)\left(\rho_{2}+\rho_{3}\right), \\
g_{11}= & \tau_{0} \bar{D} \beta\left(\bar{\rho}_{2}^{*}-1\right)\left(\operatorname{Re}\left\{\rho_{2}\right\}+\operatorname{Re}\left\{\rho_{3}\right\}\right) \\
g_{02}= & 2 \tau_{0} \bar{D} \beta\left(\bar{\rho}_{2}^{*}-1\right)\left(\bar{\rho}_{2}+\bar{\rho}_{3}\right) \\
g_{21}= & 2 \beta \tau_{0} \bar{D}\left(\bar{\rho}_{2}^{*}-1\right)\left(W_{11}^{(1)}(0) \rho_{2}+\frac{1}{2} W_{20}^{(1)}(0) \bar{\rho}_{2}+W_{11}^{(2)}(0)+\frac{1}{2} W_{20}^{(2)}(0)\right. \\
& \left.+W_{11}^{(1)}(0) \rho_{3}+\frac{1}{2} W_{20}^{(1)}(0) \bar{\rho}_{3}+W_{11}^{(3)}(0)+\frac{1}{2} W_{20}^{(3)}(0)\right)
\end{aligned}
$$

with

$$
\begin{aligned}
& W_{20}(\theta)=\frac{i g_{20} \rho(0)}{\tau_{0} \omega_{0}} e^{i \tau_{0} \omega_{0} \theta}+\frac{i \bar{g}_{02} \bar{\rho}(0)}{3 \tau_{0} \omega_{0}} e^{-i \tau_{0} \omega_{0} \theta}+E_{1} e^{2 i \tau_{0} \omega_{0} \theta}, \\
& W_{11}(\theta)=-\frac{i g_{11} \rho(0)}{\tau_{0} \omega_{0}} e^{i \tau_{0} \omega_{0} \theta}+\frac{i \bar{g}_{11} \bar{\rho}(0)}{\tau_{0} \omega_{0}} e^{-i \tau_{0} \omega_{0} \theta}+E_{2},
\end{aligned}
$$

where $E_{1}$ and $E_{2}$ can be determined by the following equations:

$$
\begin{gathered}
\left(\begin{array}{ccccc}
2 i \omega_{0}-a_{11} & -a_{12} & -a_{13} & 0 & -a_{15} \\
-a_{21} & 2 i \omega_{0}-a_{22} & -a_{23} & 0 & 0 \\
0 & -a_{32} & 2 i \omega_{0}-a_{33} e^{-2 i \tau_{0} \omega_{0}} & 0 & 0 \\
0 & 0 & -a_{43} & 2 i \omega_{0}-a_{44} & 0 \\
0 & 0 & -b_{53} e^{-2 i \tau_{0} \omega_{0}} & -b_{54} e^{-2 i \tau_{0} \omega_{0}} & 2 i \omega_{0}-a_{55}
\end{array}\right) E_{1} \\
=\left(\begin{array}{cccc}
-\beta\left(\rho_{2}+\rho_{3}\right) \\
\beta\left(\rho_{2}+\rho_{3}\right) \\
0 \\
0 \\
0
\end{array}\right), \\
\left(\begin{array}{ccccc}
a_{11} & a_{12} & a_{13} & 0 & a_{15} \\
a_{21} & a_{22} & a_{23} & 0 & 0 \\
0 & a_{32} & a_{33} & 0 & 0 \\
0 & 0 & a_{43} & a_{44} & 0 \\
0 & 0 & b_{53} & b_{54} & a_{55}
\end{array}\right) E_{2}=-\left(\begin{array}{c}
-\beta\left(\operatorname{Re}\left\{\rho_{2}\right\}+\operatorname{Re}\left\{\rho_{3}\right\}\right) \\
\beta\left(\operatorname{Re}\left\{\rho_{2}\right\}+\operatorname{Re}\left\{\rho_{3}\right\}\right) \\
0 \\
0 \\
0
\end{array}\right)
\end{gathered}
$$

Then, we can get the following coefficients:

$$
\begin{aligned}
& C_{1}(0)=\frac{i}{2 \tau_{0} \omega_{0}}\left(g_{11} g_{20}-2\left|g_{11}\right|^{2}-\frac{\left|g_{02}\right|^{2}}{3}\right)+\frac{g_{21}}{2}, \\
& \mu_{2}=-\frac{\operatorname{Re}\left\{C_{1}(0)\right\}}{\operatorname{Re}\left\{\lambda^{\prime}\left(\tau_{0}\right)\right\}} \\
& \beta_{2}=2 \operatorname{Re}\left\{C_{1}(0)\right\} \\
& T_{2}=-\frac{\operatorname{Im}\left\{C_{1}(0)\right\}+\mu_{2} \operatorname{Im}\left\{\lambda^{\prime}\left(\tau_{0}\right)\right\}}{\tau_{0} \omega_{0}}
\end{aligned}
$$


In conclusion, we have the following results.

Theorem 2 Let $E_{*}\left(S_{*}, L_{*}, B_{*}, Q_{*}, R_{*}\right)$ be the viral equilibrium of system (2).

(i) The Hopf bifurcation at the viral equilibrium $E_{*}\left(S_{*}, L_{*}, B_{*}, Q_{*}, R_{*}\right)$ is supercritical if $\mu_{2}>0$ and subcritical if $\mu_{2}<0$;

(ii) The bifurcating periodic solutions are stable if $\beta_{2}<0$ and unstable if $\beta_{2}>0$;

(iii) The period of the bifurcating periodic solutions increases if $T_{2}>0$ and decreases if $T_{2}<0$.

\section{Numerical simulations}

This section is concerned with some numerical simulations of system (2) to illustrate the results obtained in Sections 2 and 3. We fix the parameter set $\mu=0.02, \beta=0.3, \varepsilon=0.3$, $\alpha=0.3, \mu=0.35, \gamma=0.1, \eta=0.4, \sigma=0.2, \delta=0.1$ and let $\tau$ vary. With these parameter values, we get the following particular case of system (2):

$$
\left\{\begin{array}{l}
\frac{d S(t)}{d t}=0.02-0.3 S(t)(L(t)+B(t))+0.3 R(t)-0.02 S(t) \\
\frac{d L(t)}{d t}=0.3 \beta S(t)(L(t)+B(t))-0.32 L(t) \\
\frac{d B(t)}{d t}=0.3 L(t)-0.72 B(t) \\
\frac{d Q(t)}{d t}=0.1 B(t)-0.32 Q(t) \\
\frac{d R(t)}{d t}=0.1 Q(t-\tau)-0.32 R(t)+0.4 B(t-\tau) .
\end{array}\right.
$$

By direct computation we get $R_{0}=1.3281$. Then, we obtain the unique viral equilibrium $E_{*}(0.7530,0.0326,0.0136,0.0043,0.0183)$. Further, we can verify that the conditions for the occurrence of a Hopf bifurcation are satisfied. By complex computations we obtain $\omega_{0}=0.5499$ and $\tau_{0}=53.3616$. From Theorem 1 we easily see that $E_{*}(0.7530,0.0326,0.0136,0.0043,0.0183)$ is asymptotically stable for $\tau \in[0,53.3616)$, which is illustrated by Figure 1. A Hopf bifurcation occurs when $\tau=\tau_{0}=53.3616$, $E_{*}(0.7530,0.0326,0.0136,0.0043,0.0183)$ loses its stability, and a family of periodic solutions bifurcate from $E_{*}(0.7530,0.0326,0.0136,0.0043,0.0183)$. Therefore, the bifurcating periodic solutions exist at least for the values of $\tau$ larger than the critical value $\tau_{0}$. This property can be seen from Figure 2 .

Further, we obtain $\lambda^{\prime}\left(\tau_{0}\right)=0.5386-2.2362 i$ and $C_{1}(0)=-0.9466-7.6058 i$ by some complex computations. Then, we can get $\beta_{2}=-1.8932<0, \mu_{2}=1.7575>0$, and $T_{2}=$ $0.3931>0$. According to Theorem 2, we can conclude that the Hopf bifurcation is supercritical, the bifurcating periodic solutions are stable, and the period of the bifurcating periodic solutions increases. Since the bifurcating periodic solutions are stable, we know that the five kinds of computers in system (32) may coexist in an oscillatory mode from the viewpoint of biology. In this case, it is difficult to control the propagation of computer virus described in system (32).

\section{Conclusions}

A delayed SLBQRS computer virus model is proposed based on the model studied in [17]. Compared with the model studied in [17], the model in this paper incorporates the time delay due to the period that the antivirus software uses to clean the viruses in the breaking out computers and the quarantined computers. So, our delayed SLBQRS computer virus model is more general. 

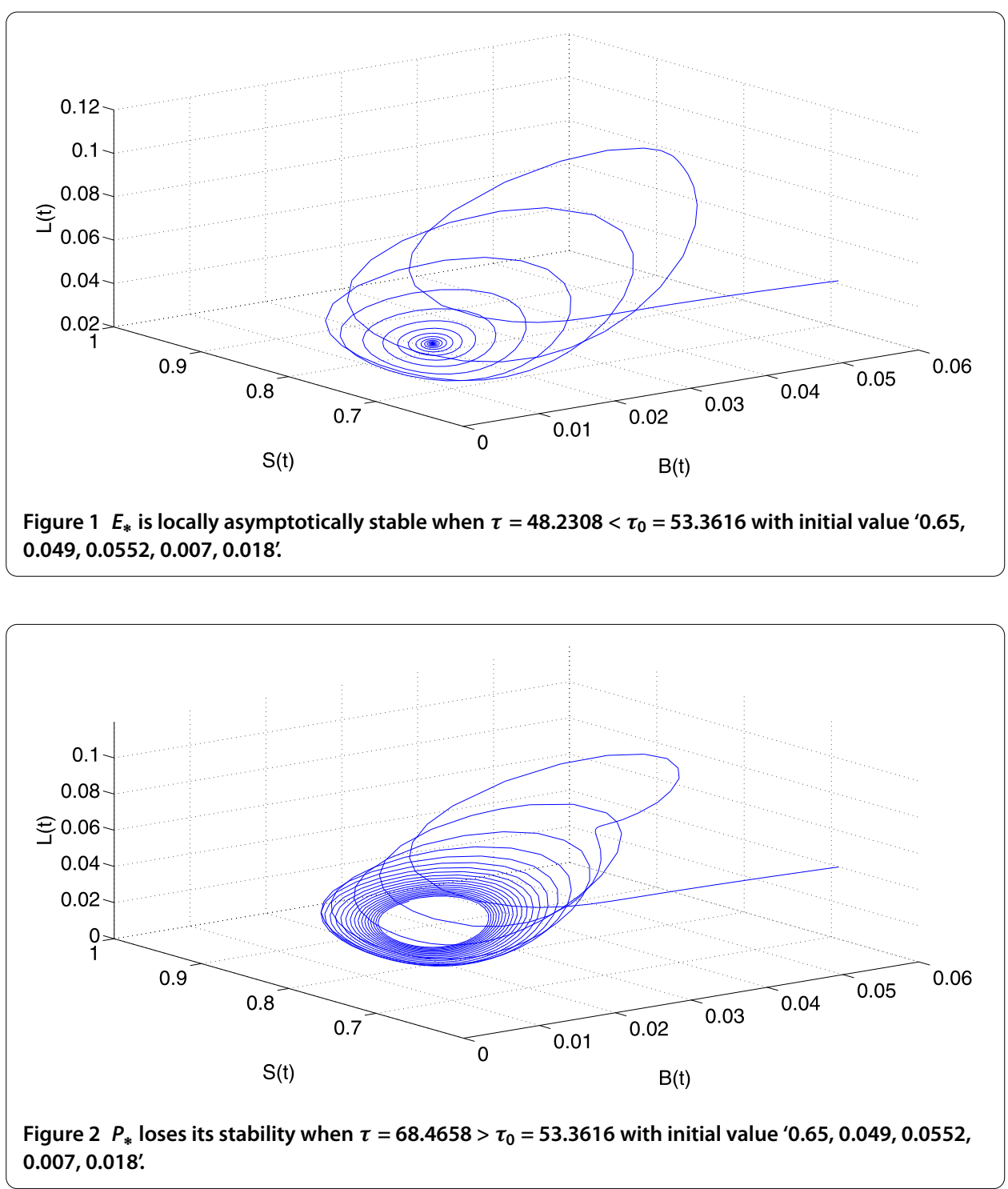

We mainly investigate the effect of the time delay on the model. We prove that there exists a critical value $\tau_{0}$ of the time delay below which the model is stable and above which the model will lose its stability. Practically speaking, propagation of the computer viruses can be controlled easily when the model is stable. However, it will be out of control when the model is unstable. That is, the time delay in system (2) can affect propagation of the computer viruses. Furthermore, properties of the Hopf bifurcation at the critical value $\tau_{0}$ are also investigated.

It should be pointed out that the delayed model in this paper only considers the time delay due to the period that the antivirus software uses to clean the viruses. There are also some other types of delay in system (2) such as latent delay and delay due to a temporary immunity period of the recovered computers. We will investigate dynamics of system (2) with multiple delays in the near future. 
Authors' contributions

All authors contributed equally to the writing of this paper. All authors read and approved the final manuscript.

\section{Acknowledgements}

The authors would like to thank the editor and anonymous referees for their constructive suggestions on improving the presentation of the paper. This work was supported by Natural Science Foundation of the Higher Education Institutions of Anhui Province (No. KJ2015A144) and Anhui Provincial Natural Science Foundation (Nos. 1608085QF151, 1608085QF145).

Received: 9 October 2016 Accepted: 22 December 2016 Published online: 27 January 2017

\section{References}

1. Kumar, M, Mishar, BK, Panda, TC: Effect of quarantine and vaccination on infectious nodes in computer network. Int. J. Comput. Netw. Appl. 2, 92-98 (2015)

2. Szor, P: The Art of Computer Virus Research and Defense. Addison-Wesley, Reading (2005)

3. Kephart, JO, White, SR: Directed-graph epidemiological models of computer viruses. In: Proc. 1991 IEEE Comput. Society Symp. Res. Secur. Privacy, pp. 343-359 (1991)

4. Piqueria, JRC: A modified epidemiological model for computer viruses. Appl. Math. Comput. 213, 355-360 (2009)

5. Piqueira, JRC, Cesar, FB: Dynamical models for computer virus propagation. Math. Probl. Eng. 2008, Article ID 940526 (2008)

6. Keeling, MJ, Eames, KTD: Network and epidemic models. J. R. Soc. Interface 2, 295-307 (2005)

7. Piqueira, JRC, Navarro, BF, Monteiro, LHA: Epidemiological models applied to virus in computer network. J. Comput. Sci. 1, 31-34 (2005)

8. Yuan, YH, Chen, G: Network virus epidemic model with the point-to-group information propagation. Appl. Math. Comput. 206, 357-367 (2008)

9. Dong, T, Liao, XF, Li, HQ: Stability and Hopf bifurcation in a computer virus model with multistate antivirus. Abstr. Appl. Anal. 2012, Article ID 841987 (2012)

10. Peng, M, He, X, Huang, JJ, Dong, T: Modeling computer virus and its dynamics. Math. Probl. Eng. 2013, Article ID $842614(2013)$

11. Peng, M, Mou, HJ: A novel computer virus model and its stability. J. Netw. 9, 367-374 (2014)

12. Mishra, BK, Saini, DK: SEIRS epidemic model with delay for transmission of malicious objects in computer network. Appl. Math. Comput. 188, 1476-1482 (2007)

13. Zhang, ZZ, Yang, HZ: Hopf bifurcation analysis for a computer virus model with two delays. Abstr. Appl. Anal. 2013, Article ID 560804 (2013)

14. Mishra, BK, Pandey, SK: Dynamic model of worms with vertical transmission in computer network. Appl. Math. Comput. 217, 8438-8446 (2011)

15. Mishra, BK, Jha, N: SEIQRS model for the transmission of malicious objects in computer network. Appl. Math. Model. 34, 710-715 (2010)

16. Yang, LX, Yang, XF: A new epidemic model of computer viruses. Commun. Nonlinear Sci. Numer. Simul. 19, 1935-1944 (2014)

17. Kumar, M, Mishar, BK, Panda, TC: Stability analysis of a quarantined epidemic model with latent and breaking-out over the Internet. Int. J. Hybrid Inf. Technol. 8, 133-148 (2015)

18. Yang, M, Zhang, Z, Li, Q, Zhang, G: An SLBRS model with vertical transmission of computer virus over the Internet. Discrete Dyn. Nat. Soc. 2012, Article ID 925648 (2012)

19. Hassard, BD, Kazarinoff, ND, Wan, YH: Theory and Applications of Hopf Bifurcation. Cambridge University Press, Cambridge (1981)

20. Zhang, TL, Jiang, HJ, Teng, ZD: On the distribution of the roots of a fifth degree exponential polynomial with application to a delayed neural network model. Neurocomputing 72, 1098-1104 (2009)

21. Bianca, C, Ferrara, M, Guerrini, L: The cai model with time delay: existence of periodic solutions and asymptotic analysis. Appl. Math. Inf. Sci. 7, 21-27 (2013)

22. Bianca, C, Ferrara, M, Guerrini, L: The time delays' effects on the qualitative behavior of an economic growth model. Abstr. Appl. Anal. 2013, Article ID 901014 (2013)

23. Bianca, C, Guerrini, L: Existence of limit cycles in the Solow model with delayed-logistic population growth. Sci. World J. 2014, Article ID 207806 (2014)

\section{Submit your manuscript to a SpringerOpen ${ }^{\ominus}$ journal and benefit from:}

- Convenient online submission

- Rigorous peer review

- Immediate publication on acceptance

Open access: articles freely available online

- High visibility within the field

- Retaining the copyright to your article

Submit your next manuscript at $>$ springeropen.com 